\title{
Comparative morphometric evaluation of hepatic hemosiderosis in wild Magellanic penguins (Spheniscus magellanicus) infected with different Plasmodium spp. subgenera
}

Avaliação morfométrica comparativa da hemossiderose hepática em pinguins-de-Magalhães (Spheniscus magellanicus) de vida livre infectados por diferentes subgêneros de Plasmodium spp.

Ana Carolina Ewbank ${ }^{1 *}$ (1); Ricardo de Francisco Strefezzi²; Carlos Sacristán ${ }^{1}$; Cristiane Kiyomi Miyaji Kolesnikovas ${ }^{3}$; Aryse Martins ${ }^{4}$; Luis Felipe Silva Pereira Mayorga ${ }^{5}$; Ralph Eric Thijl Vanstreels ${ }^{1}$; José Luiz Catão-Dias ${ }^{1}$

\author{
${ }^{1}$ Laboratório de Patologia Comparada de Animais Selvagens, Departamento de Patologia, Universidade de Sáo Paulo - USP, \\ São Paulo, SP, Brasil \\ ${ }^{2}$ Laboratório de Oncologia Comparada e Translacional, Faculdade de Zootecnia e Engenharia de Alimentos, Universidade de São \\ Paulo - USP, Pirassununga, SP, Brasil \\ ${ }^{3}$ Associação R3 Animal, Florianópolis, SC, Brasil \\ ${ }^{4}$ Centro de Recuperação de Animais Marinhos - CRAM, Museu Oceanográfico Professor Eliézer de Carvalho Rios, Universidade \\ Federal do Rio Grande - FURG, Rio Grande, RS, Brasil \\ ${ }^{5}$ Instituto de Pesquisa e Reabilitação de Animais Marinhos - IPRAM, Cariacica, ES, Brasil
}

Received: 5 August, 2018

Accepted: 12 November, 2018

\begin{abstract}
Avian malaria is one of the most important diseases of captive penguins. We employed morphometric techniques to evaluate hepatic hemosiderosis in rehabilitating wild Magellanic penguins (Spheniscus magellanicus) that were negative $(\mathrm{n}=9)$ or naturally infected by different subgenera of Plasmodium spp. $(\mathrm{n}=24)$, according with: Plasmodium subgenera (Haemamoeba, Huffia, Other lineages, and Unidentified lineages), severity of Plasmodium histopathological lesions, and concurrent diseases, age class (juvenile or adult plumage), sex (male, female or not determined), body score (emaciated, thin, good, excellent, not available), molt, presence or absence of oil contamination upon admission, iron supplementation, and rehabilitation center. The percentage of the area occupied by hemosiderin was called 'Index of Hepatic Hemosiderosis (IHH)'. Plasmodium-positive females presented significantly higher IHH values $(17.53 \pm 12.95 \%)$ than males $(7.20 \pm 4.25 \% ; \mathrm{p}=0.041)$. We observed higher levels of congestion $(\mathrm{p}=0.0182)$ and pneumonia $(\mathrm{p}=0.0250)$ severity between Unidentified lineages vs. Huffia. We believe that the hepatic hemosiderosis observed in this study was multifactorial, the result of pathological processes caused by malaria, molting, hemoglobin and myoglobin catabolism during migration, anemia, concomitant diseases, and iron supplementation, all possibly potentiated by decreased liver mass. Further studies are needed to clarify the mechanisms of these hypotheses.
\end{abstract}

Keywords: Avian malaria, iron, hemosiderin, liver, morphometry, penguin.

\section{Resumo}

Malária aviária é uma das mais relevantes doenças em pinguins cativos. Foram aplicadas técnicas morfométricas para avaliar a hemossiderose hepática em pinguins-de-Magalhães (Spheniscus magellanicus) de vida livre em reabilitação negativos $(\mathrm{n}=9)$ e naturalmente infectados por diferentes subgêneros de Plasmodium spp. ( $\mathrm{n}=24)$, quanto a: subgênero de Plasmodium (Haemamoeba, Huffia, Outras Linhagens, e Linhagens não identificadas), severidade das lesóes histopatológicas causadas por Plasmodium e doenças concomitantes, faixa etária (plumagem juvenil ou adulta), sexo (macho, fêmea, indeterminado), condição corporal (emaciado, magro, bom, excelente, indisponível), muda, presença/ausência de óleo a admissão,

*Corresponding author: Ana Carolina Ewbank. Laboratório de Patologia Comparada de Animais Selvagens, Departamento de Patologia, Universidade de São Paulo - USP, Av. Orlando Marques de Paiva, 87, Butantá,

CEP 05508-270, São Paulo, SP, Brasil. e-mail: carolewbank@yahoo.com.br 
suplementação de ferro, e centro de reabilitação. A porcentagem da área ocupada por hemossiderina foi denominada "Índice de Hemossiderose Hepática (IHH)". Fêmeas Plasmodium-positivas apresentaram IHH significativamente mais elevado que machos, respectivamente, $17,53 \pm 12,95 \%$ e 7,20 \pm 4,25\% ( $\mathrm{p}=0,041)$. Níveis mais elevados de congestáo ( $\mathrm{p}=0,0182)$ e pneumonia ( $\mathrm{p}=0,0250)$ foram observados entre Linhagens não identificadas vs. Huffia. Possivelmente, a hemossiderose hepática observada nesse estudo seja multifatorial, resultado de processos patológicos causados por malária, muda, catabolismo de hemoglobina e mioglobina durante a migração, anemia, doenças concomitantes e suplementação de ferro, potencialmente intensificados por massa hepática reduzida. Estudos complementares são necessários para esclarecer os mecanismos de tais hipóteses.

Palavras-chave: Malária aviária, ferro, hemosiderina, fígado, morfometria, pinguim.

\section{Introduction}

The Magellanic penguin (Spheniscus magellanicus) breeds in colonies located on the coasts of Chile, Argentina, and the Falkland (Malvinas) Islands. During their winter migration, individuals from the Atlantic Ocean colonies migrate along the continental shelf of northern Argentina, Uruguay, and southern Brazil (STOKES et al., 2014), where they face many challenges (PETRY \& FONSECA, 2002; GARCÍA-BORBOROGLU et al., 2006; BRANDĀO et al., 2011; BALDASSIN et al., 2012; REZENDE et al., 2013; KEHRIG et al., 2015). Birds stranded alive are rescued and referred to local rehabilitation centers along the Brazilian coast (GARCÍA-BORBOROGLU et al., 2010; RODRIGUES et al., 2010; RUOPPOLO et al., 2012)

While under rehabilitation, these birds may be exposed to avian malaria, one of the most significant threats to the conservation of penguins, able to seriously compromise their survival in captivity (JONES \& SHELLAM, 1999; VANSTREELS et al., 2016). Avian malaria is transmitted by Culicidae mosquitoes infected with Plasmodium spp. protozoans (VALKIŪNAS, 2005), and is relatively asymptomatic in most avian species, but potentially pathogenic for those species that have not co-evolved with the parasite, such as penguins (Spheniscidae) (VALKIŪNAS, 2005; VANSTREELS et al., 2016). Penguins are highly susceptible to avian malaria. Rapid and severe outbreaks with high mortality (VANSTREELS et al., 2016) have been reported worldwide in populations of several penguin species in the wild (FANTHAM \& PORTER, 1944; LAIRD, 1950; LEVIN et al., 2009), in captivity (GRINER \& SHERIDAN, 1967; BAK et al., 1984; FIX et al., 1988), and undergoing rehabilitation (PARSONS \& UNDERHILL, 2005). In Brazil, reports of avian malaria in Magellanic penguins include birds in zoos (BUENO et al., 2010) and rehabilitation centers (SILVEIRA et al., 2013; VANSTREELS et al., 2014, 2015).

Iron homeostasis in birds, as in other vertebrates, is regulated mainly through intestinal absorption and excess iron is stored as intracellular aggregations of ferritin or hemosiderin, particularly in hepatic and splenic cells (NORAMBUENA \& BOZINOVIC, 2009). Hemosiderosis is the accumulation of iron in hepatic and splenic sinusoidal macrophages and hepatocytes without significant architectural, cellular, or functional alterations (LOWENSTEIN \& MUNSON, 1999). Iron absorption is influenced by age, iron and health status, gastrointestinal conditions, the amount and chemical form of the iron ingested, and the amount and proportions of various other organic and inorganic components of the diet (CRISSEY et al., 2000; SHEPPARD \& DIERENFELD, 2002; PEREIRA et al., 2010). Although there is no conclusive evidence that hepatic hemosiderosis has any clinical significance in birds, it has been associated with concurrent infections, neoplasia, parasitism, anemia, hepatopathies, intoxications, starvation, oil exposure, trauma, lead poisoning, and the physiological cycle (e.g., egg production and laying, migration, and molting) (CORK et al., 1995; GOTTDENKER et al., 2008; PEREIRA et al., 2010; KLASING et al., 2012).

Hemosiderosis, especially hepatic, has been previously reported in Plasmodium-infected penguins (FIX et al., 1988; GRILO et al., 2016), believed to be caused by the build up of hemosiderin in visceral macrophages following intravascular hemolysis and phagocytosis of parasitized cells (VANSTREELS \& PARSONS, 2014). However, these studies did not evaluate if such condition varied among Plasmodium spp. subgenera or considered other possible causes of hemosiderosis aside from Plasmodium spp. infection. Considering the importance of avian malaria to penguin conservation and the limited amount of information regarding iron metabolism and the pathogenesis of avian malaria in birds, our objective was to quantify the hepatic hemosiderosis in Plasmodium spp.- negative and - naturally infected Magellanic penguins that died while under care in rehabilitation centers in Brazil, in regards to the presence of Plasmodium and Plasmodium species/lineages, and within the context of these birds' biology and individual history.

\section{Material and Methods}

The Magellanic penguins evaluated in this study represent all Plasmodium-positive ( $\mathrm{n}=24)$ and some of the Plasmodium-negative $(n=9)$ birds, that died while under rehabilitation along $2500 \mathrm{~km}$ of the Brazilian coastline between January 2009 and February 2013 during studies on the prevalence of Plasmodium in Magellanic penguins (SILVEIRA et al., 2013; VANSTREELS et al., 2014, 2015). Physical and postmortem examinations, including necropsy and hematology findings, are described elsewhere (VANSTREELS et al., 2015). The positive group comprised 24 Plasmodium-positive animals: 21 individuals naturally infected by Plasmodium spp., previously diagnosed by morphologic (blood smears, histopathology) and molecular techniques (nested PCR, genetic sequencing) (VANSTREELS et al., 2015); and 3 additional natural infection cases identified using the same methods (cases 3, 14, and 15). Positive birds were divided into 4 study groups: (1) Haemamoeba (P. cathemerium and P. tejerai), (2) Huffia (P. elongatum), (3) Other lineages (P. nucleophilum, P. unalis, and Plasmodium sp. lineages E, $\mathrm{G}$, and $\mathrm{H}$ ), and (4) Unidentified lineages (Plasmodium sp. that 
could not be morphologically or molecularly characterized). The positive birds evaluated in this study either (a) died suddenly, (b) presented antemortem malaria-associated clinical signs, dying after unsuccessful treatment, or (c) were euthanized for reasons unrelated to malaria (e.g., aspergillosis). Nine Plasmodium-negative Magellanic penguins were selected based on individual history and a negative diagnosis for Plasmodium spp. on both nested PCR and blood smears. Selected Plasmodium-negative birds died without presenting any clinical signs of concurrent infections, and remained in captivity for similar periods as the positive birds. All birds from both groups (positive and negative) were kept under similar infrastructure and husbandry conditions, and diet and iron supplementation varied among rehabilitation facilities (Table 1).

All birds were examined postmortem, and liver samples were collected and fixed in $10 \%$ formalin for a minimum of 72 hours before processing. Paraffin embedded liver fragments were sectioned at $5 \mu \mathrm{m}$ and stained with haematoxylin-eosin and Perls stains, as described by Luna (1992) (Figure 1). The Index of Hepatic

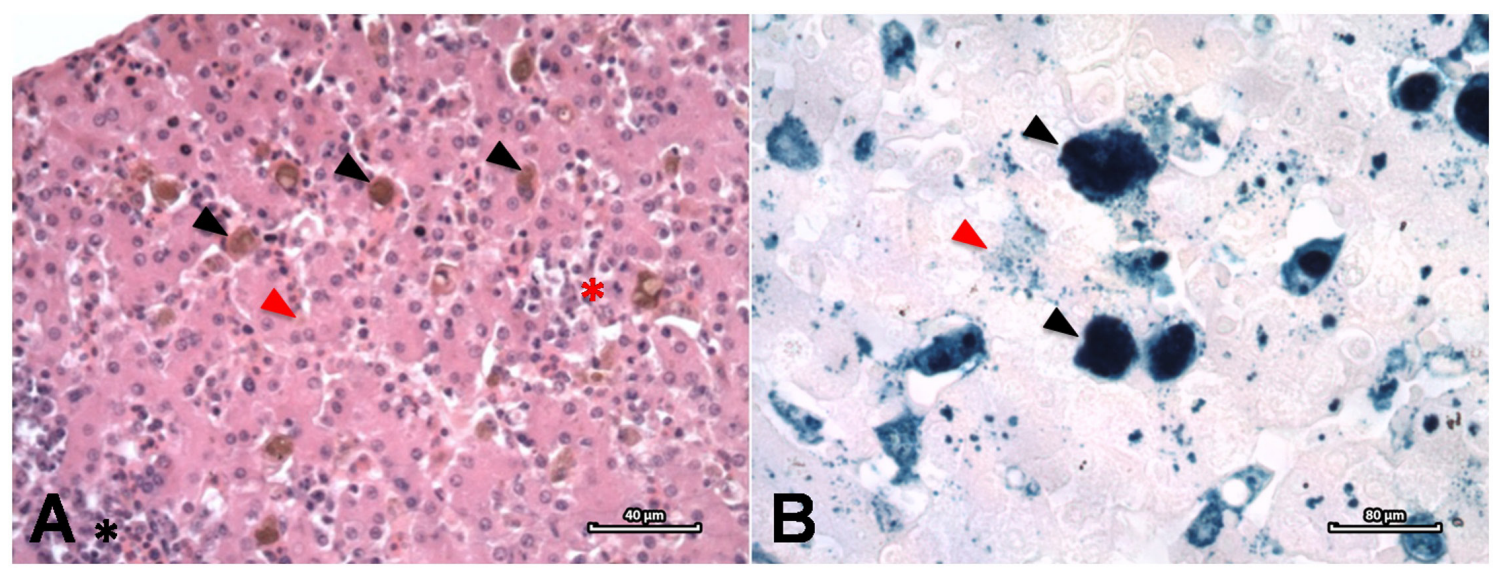

Figure 1. Hepatic hemosiderosis in a Magellanic penguin (Spheniscus magellanicus). A. Areas of intracellular hemosiderin deposition in Kupffer cells (black arrowheads) and hepatocytes (red arrowhead), diffuse cordonal derangement (red asterisk) and inflammatory infiltrate (black asterisk). H\&E stain. $40 \mu \mathrm{m}$. B. Intracellular hemosiderin deposits within Kupffer cells (black arrowheads) and hepatocytes (red arrow). Perls stain. $80 \mu \mathrm{m}$.

Table 1. Plasmodium-positive and control Magellanic penguins (Spheniscus magellanicus) according with rehabilitation center, iron supplementation, administration criteria and diet.

\begin{tabular}{|c|c|c|c|}
\hline \multirow{2}{*}{$\begin{array}{l}\text { Rehabilitation } \\
\text { Center }\end{array}$} & Iron supplementation & \multirow{2}{*}{ Administration Criteria } & Diet \\
\hline & (Dose/Route/Frequency) & & (whole fish-based) \\
\hline \multirow[t]{3}{*}{ CRAM $^{a}$} & $\begin{array}{l}\text { Ferrodex }{ }^{\mathrm{d}} \rightarrow 0.2 \mathrm{mg} / \mathrm{kg} / \mathrm{IM} \text { - single dose } \\
(\text { until 2013) }\end{array}$ & Hematocrit $<35 \%$ & $\begin{array}{c}\text { banded croaker } \\
\text { (Paralonchurus brasiliensis) }\end{array}$ \\
\hline & $\begin{array}{l}\text { Ferrodex } \rightarrow 0.2 \mathrm{mg} / \mathrm{kg} / \mathrm{IM}-\text { single dose } \\
\text { (from } 2013 \text { to the present) }\end{array}$ & $\begin{array}{l}\text { Hematocrit }<30 \% \text { until the parameter reaches } \\
\text { the acceptable interval for the species }\end{array}$ & $\begin{array}{l}\text { stripped weakfish } \\
\text { (Cynoscion guatucupa) } \\
\text { acoupa weakfish } \\
\text { (Cynoscion acoupa) }\end{array}$ \\
\hline & $\begin{array}{l}\text { Ferrodex } \rightarrow 0.2 \mathrm{mg} / \mathrm{kg} / \mathrm{IM}-\text { single dose in } \\
\text { association with Hemolitan pet }{ }^{e} \rightarrow 0.1 \mathrm{mg} / \mathrm{kg}\end{array}$ & $\begin{array}{l}\text { Hematocrit }<35 \% \text { until the parameter reaches } \\
\text { the acceptable interval for the species }\end{array}$ & $\begin{array}{l}\text { Argentine croaker } \\
\text { (Umbrina canosai) }\end{array}$ \\
\hline
\end{tabular}
$\mathrm{PO}-\mathrm{q} 24 \mathrm{~h}$

(from 2013 to the present)

whitemouth croaker (Micropogonias furnieri)
R3 Animal ${ }^{\mathrm{b}} \quad$ Ferrodex $\rightarrow 1 \mathrm{ml} / \mathrm{IM}$ - single dose Upon admission

IPRAM $^{c}$
Hemolitan pet $\rightarrow 1 \mathrm{ml} / \mathrm{PO}-\mathrm{q} 24 \mathrm{~h}$
Penguins treated as huddles. Medication administered upon admission and maintained for days to weeks, until the birds exhibited normal colored oral mucosae

${ }^{\mathrm{a}}$ CRAM = Centro de Recuperação de Animais Marinhos (Rio Grande do Sul state, Brazil). ${ }^{\mathrm{b}} \mathrm{R} 3$ = Associação R3 Animal (Santa Catarina state, Brazil). ${ }^{\mathrm{c} I P R A M}=$ Instituto de Pesquisa e Reabilitação de Animais Marinhos (Espírito Santo state, Brazil). ${ }^{\mathrm{d} F e r r o d e x}{ }^{\circledR}$ (Tortuga, Companhia Zootécnica Agrária, São Amaro, São Paulo, Brazil). ${ }^{e}$ Hemolitan

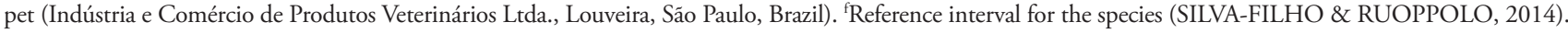


Hemosiderosis $(\mathrm{IHH})$ was evaluated according with the presence of infection, Plasmodium species/lineage, malaria severity (based on the classification of the histopathological findings observed in heart, lung, liver, and spleen samples: mild, mild to moderate, moderate, moderate to severe, and severe - Table 2), concurrent diseases and/or significant lesions, individual history (age class

Table 2. Plasmodium-positive and control Magellanic penguins according with the Index of Hepatic Hemosiderosis (IHH), study group, Plasmodium lineage, Plasmodium species and malaria severity.

\begin{tabular}{|c|c|c|c|c|}
\hline Case & Study group & Plasmodium lineage & $\begin{array}{c}\text { IHH } \\
(\%)\end{array}$ & Malaria severity \\
\hline \multirow{3}{*}{1} & \multirow{3}{*}{ Haemamoeba } & \multirow{3}{*}{ P. cathemerium } & \multirow{3}{*}{0.4} & Moderate pulmonary congestion \\
\hline & & & & Moderate granulocytic pneumonia \\
\hline & & & & Moderate-severe monocytic hepatitis \\
\hline \multirow{4}{*}{2} & \multirow{4}{*}{ Haemamoeba } & \multirow{4}{*}{ P. cathemerium } & \multirow{4}{*}{14.8} & Moderate-severe pulmonary congestion \\
\hline & & & & Moderate to severe granulocytic pneumonia \\
\hline & & & & Mixed moderate-severe splenitis \\
\hline & & & & Mild-moderate monocytic hepatitis \\
\hline \multirow{9}{*}{3} & \multirow{9}{*}{ Haemamoeba } & \multirow{9}{*}{ P. cathemerium } & \multirow{9}{*}{1.1} & Mild-moderate acute diffuse granulocytic pneumonia \\
\hline & & & & Parasitic pneumonia compatible with Schistosoma sp. \\
\hline & & & & Diffuse necrotic splenitis \\
\hline & & & & Splenic limphocytolysis, histiocytolysis and lymphoid hypoplasia \\
\hline & & & & Parasitic granulomatous hepatitis \\
\hline & & & & Diffuse renal degeneration \\
\hline & & & & Mild cerebellar congestion \\
\hline & & & & Mild necrotic focal myocarditis \\
\hline & & & & Mild monocytic diffuse myocarditis \\
\hline \multirow{4}{*}{4} & \multirow{4}{*}{ Haemamoeba } & \multirow{4}{*}{ P. cathemerium } & \multirow{4}{*}{18.3} & Moderate pulmonary congestion \\
\hline & & & & Moderate granulocytic pneumonia \\
\hline & & & & Moderate-severe granulocytic splenitis \\
\hline & & & & Moderate mixed hepatitis \\
\hline \multirow{4}{*}{5} & \multirow{4}{*}{ Haemamoeba } & \multirow{4}{*}{ P. cathemerium } & \multirow{4}{*}{4.2} & Moderate-severe pulmonary congestion \\
\hline & & & & Mild granulocytic pneumonia \\
\hline & & & & Moderate-severe mixed splenitis \\
\hline & & & & Moderate-severe monocytic hepatitis \\
\hline \multirow{4}{*}{6} & \multirow{4}{*}{ Haemamoeba } & \multirow{4}{*}{ P. cathemerium } & & Moderate pulmonary congestion \\
\hline & & & 58 & Moderate granulocytic pneumonia \\
\hline & & & 5.8 & Mild-moderate granulocytic splenitis \\
\hline & & & & Mild-moderate mixed hepatitis \\
\hline & & & & Moderate-severe pulmonary congestion \\
\hline & & & & Moderate granulocytic pneumonia \\
\hline 7 & Haemamoeba & P. tejerai & 10.8 & Mild-moderate granulocytic splenitis \\
\hline & & & & Moderate monocytic hepatitis \\
\hline & & & & Hepatic ductal hyperplasia \\
\hline & & & & Moderate-severe pulmonary congestion \\
\hline 8 & Haemamoeba & P. tejerai & 1.5 & Moderate mixed hepatitis \\
\hline & & & & Hepatic ductal hyperplasia \\
\hline & & & & Moderate pulmonary congestion \\
\hline & & & & Moderate granulocytic pneumonia \\
\hline 9 & Haemamoeba & P. tejerai & 38.2 & Mild-moderate granulocytic splenitis \\
\hline & & & & Mild-moderate granulocytic hepatitis \\
\hline & & & & Hepatic ductal hyperplasia \\
\hline & & & & Moderate-severe pulmonary congestion \\
\hline & & & & Severe granulocytic pneumonia \\
\hline 10 & Hasmamash & D toiergi & 310 & Mild-moderate mixed splenitis \\
\hline 10 & Паетатоева & 1. rejerai & 31.9 & Moderate monocytic hepatitis \\
\hline & & & & Hepatic ductal hyperplasia \\
\hline & & & & Mild monocytic myocarditis \\
\hline
\end{tabular}


Table 2. Continued...

\begin{tabular}{|c|c|c|c|c|}
\hline Case & Study group & Plasmodium lineage & $\begin{array}{c}\text { IHH } \\
(\%)\end{array}$ & Malaria severity \\
\hline \multirow{3}{*}{11} & \multirow{3}{*}{ Haemamoeba } & \multirow{3}{*}{ P. tejerai } & \multirow{3}{*}{9.4} & Mild-moderate pulmonary congestion \\
\hline & & & & $\begin{array}{l}\text { Mild-moderate granulocytic pneumonia } \\
\text { Mild-moderate mixed splenitis }\end{array}$ \\
\hline & & & & Mild granulocytic myocarditis \\
\hline \multirow{3}{*}{12} & \multirow{3}{*}{ Huffia } & \multirow{3}{*}{ P. elongatum } & \multirow{3}{*}{22.8} & Moderate pulmonary congestion \\
\hline & & & & Mild granulocytic pneumonia \\
\hline & & & & Moderate monocytic hepatitis \\
\hline \multirow{9}{*}{13} & \multirow{9}{*}{ Huffia } & \multirow{9}{*}{ P. elongatum } & \multirow{9}{*}{1.4} & Mild-moderate congestion \\
\hline & & & & Acute granulocytic diffuse myocarditis \\
\hline & & & & Moderate cerebellar congestion \\
\hline & & & & Moderate diffuse acute granulocytic pneumonia \\
\hline & & & & $\begin{array}{l}\text { Moderate coalescent multifocal histiocytosis, moderate lymphoid hypoplasia, } \\
\text { lymphocytolisis, extramedullary erythropoiesis, mild-moderate mixed diffuse } \\
\text { necrotic splenitis }\end{array}$ \\
\hline & & & & Mixed multifocal interstitial nephritis \\
\hline & & & & Moderate-severe multifocal necrotic hepatitis \\
\hline & & & & Extramedullary hepatic erythropoiesis \\
\hline & & & & Hepatic ductal hyperplasia \\
\hline \multirow{8}{*}{14} & \multirow{8}{*}{ Huffia } & \multirow{8}{*}{ P. elongatum } & \multirow{8}{*}{6.9} & Mild cardiac congestion \\
\hline & & & & Mild acute diffuse myocarditis \\
\hline & & & & Mild to moderate cerebellar congestion \\
\hline & & & & Moderate mixed diffuse pneumonia \\
\hline & & & & Moderate pulmonary congestion \\
\hline & & & & Spleen tissue was not available for examination \\
\hline & & & & Diffuse renal degeneration \\
\hline & & & & $\begin{array}{l}\text { Moderate-severe mixed coalescent multifocal necrotic periportal hepatitis and } \\
\text { mild extramedullary erythropoiesis }\end{array}$ \\
\hline \multirow{4}{*}{15} & \multirow{4}{*}{ Other lineages } & \multirow{4}{*}{ P. nucleophilum } & \multirow{4}{*}{27.6} & Mild pulmonary congestion \\
\hline & & & & Mild-moderate monocytic pneumonia \\
\hline & & & & Mild-moderate monocytic splenitis \\
\hline & & & & Moderate-severe mixed hepatitis \\
\hline \multirow{3}{*}{16} & \multirow{3}{*}{ Other lineages } & \multirow{3}{*}{ P. unalis } & & Moderate-severe pulmonary congestion \\
\hline & & & 15 & Moderate-severe monocytic pneumonia \\
\hline & & & & Mild monocytic hepatitis \\
\hline & & & & Moderate pulmonary congestion \\
\hline & & & & Moderate granulocytic pneumonia \\
\hline 17 & Other lineages & Plasmodium sp. E & 6.2 & Moderate granulocytic splenitis \\
\hline & & & & Mild monocytic hepatitis \\
\hline & & & & Hepatic ductal hyperplasia \\
\hline & & & & Moderate-severe pulmonary congestion \\
\hline 18 & Other lineages & Plasmodium sn $G$ & 48 & Moderate granulocytic pneumonia \\
\hline 10 & Uner inneages & 1'tasmoazum sp. G & 4.0 & Moderate granulocytic splenitis \\
\hline & & & & Moderate-severe monocytic hepatitis \\
\hline & & & & Moderate-severe pulmonary congestion \\
\hline & & & & Severe granulocytic pneumonia \\
\hline 19 & Other lineages & Plasmodium sp. $G$ & 45.4 & Mild granulocytic splenitis \\
\hline & & & & Mild-moderate granulocytic hepatitis \\
\hline & & & & Severe myocardial necrosis \\
\hline & & & & Moderate pulmonary congestion \\
\hline 20 & Other lineages & Plasmodium sp. $H$ & 14.6 & Moderate mixed pneumonia \\
\hline & & & & Moderate monocytic hepatitis \\
\hline
\end{tabular}


Table 2. Continued...

\begin{tabular}{|c|c|c|c|c|}
\hline Case & Study group & Plasmodium lineage & $\begin{array}{c}\text { IHH } \\
(\%)\end{array}$ & Malaria severity \\
\hline \multirow{5}{*}{21} & \multirow{5}{*}{ Unidentified lineages } & \multirow{5}{*}{ Plasmodium sp. } & \multirow{5}{*}{10.7} & Moderate-severe pulmonary congestion \\
\hline & & & & Moderate-severe monocytic pneumonia \\
\hline & & & & Moderate granulocytic splenitis \\
\hline & & & & Moderate-severe monocytic hepatitis \\
\hline & & & & Mild-moderate monocytic myocarditis \\
\hline \multirow{5}{*}{22} & \multirow{5}{*}{ Unidentified lineages } & \multirow{5}{*}{ Plasmodium sp. } & \multirow{5}{*}{12.8} & Moderate-severe pulmonary congestion \\
\hline & & & & Moderate-severe granulocytic pneumonia \\
\hline & & & & Moderate-severe granulocytic splenitis \\
\hline & & & & Moderate granulocytic hepatitis \\
\hline & & & & Mild granulocytic hepatitis \\
\hline \multirow{5}{*}{23} & \multirow{5}{*}{ Unidentified lineages } & \multirow{5}{*}{ Plasmodium sp. } & \multirow{5}{*}{8.3} & Severe pulmonary congestion \\
\hline & & & & Severe monocytic pneumonia \\
\hline & & & & Mild-moderate mixed splenitis \\
\hline & & & & Moderate monocytic hepatitis \\
\hline & & & & Mild granulocytic myocarditis \\
\hline \multirow{4}{*}{24} & \multirow{4}{*}{ Unidentified lineages } & \multirow{4}{*}{ Plasmodium sp. } & \multirow{4}{*}{13.8} & Moderate-severe pulmonary congestion \\
\hline & & & & Moderate granulocytic pneumonia \\
\hline & & & & Mild-moderate monocytic hepatitis \\
\hline & & & & Hepatic ductal hyperplasia \\
\hline 25 & Plasmodium - negative & Negative & 0.9 & - \\
\hline 26 & Plasmodium - negative & Negative & 18.1 & - \\
\hline 27 & Plasmodium - negative & Negative & 11.6 & - \\
\hline 28 & Plasmodium - negative & Negative & 2.9 & - \\
\hline 29 & Plasmodium - negative & Negative & 0.9 & - \\
\hline 30 & Plasmodium - negative & Negative & 5.6 & - \\
\hline 31 & Plasmodium - negative & Negative & 13.4 & - \\
\hline 32 & Plasmodium - negative & Negative & 6.5 & - \\
\hline 33 & Plasmodium - negative & Negative & 12.5 & - \\
\hline
\end{tabular}

[juvenile or adult plumage] (SILVA-FILHO \& RUOPPOLO, 2014), sex [male, female or unknown], body mass on admission (emaciated, thin, good, excellent, not available [N/A]), body score, hematocrit, presence or absence of oil upon admission [oiling], molt and iron supplementation [Fe]) (Table 3). To establish the $\mathrm{IHH}$, slides were evaluated by light microscopy and captured images where analyzed by a computerized image analyses software (Image Pro ${ }^{\circledR}$ Plus, version 5.1.2.59, Media Cybernetics) to determine the percentage of liver tissue occupied by hemosiderin pigment. The intersection of the two longest axis of each bird's hepatic section was determined as the center of the sample, and used as the reference field to capture images at 20X magnification. Another 8 images were captured as TIFF files, at $50 \mu \mathrm{m}$ of this point, and $45^{\circ}$ intervals, under the same lighting conditions and magnification. The malarial pigment, called hemozoin, stains brown with Perls stain, while hemosiderin stains bright turquoise blue, promoting an easy visual distinction between the two pigments. Hemosiderin deposits were semi-automatically delineated on the Perls stained slides, starting at the lowest blue intensity to the most intense, to avoid the inclusion of artifacts and the background. When overlapping areas of formolic pigment and hemosiderin deposition where present, the areas of formolic pigment deposition where individually marked and deducted from such areas. In order to standardize the technique, a maximum digital zoom of $50 \%$ was stipulated. The average of the 9 microscopic fields was considered the IHH.

One-way ANOVA/Kruskal-Wallis test followed by Dunn's multiple comparisons test and Mann-Whitney tests were used to verify differences between the evaluated parameters and groups. The Spearman test was used to evaluate the correlation between quantitative variables. The significance level was defined as 5\%. Data were analyzed with GraphPad Prism software (Version 4.02 for Windows, GraphPad Software, Inc.).

All samples used in this study were collected in full compliance with specific federal permits issued by the Brazilian Ministry of Environment and approved by the Biodiversity Information and Authorization System (SISBIO 20825-8). All procedures were performed according to the Ethical Committee in Animal Research of the School of Veterinary Medicine and Animal Sciences, University of São Paulo (Process numbers 1757/2009 and 9411100414). 
Table 3. Plasmodium-positive and control Magellanic penguins according with the Index of Hepatic Hemosiderosis (IHH), rehabilitation center, age class, sex, body mass on admission (g), body score, hematocrit (Ht), presence or absence of oil upon admission (oil), moult, iron supplementation $(\mathrm{Fe})$ and concurrent disease and/or significant lesion.

\begin{tabular}{|c|c|c|c|c|c|c|c|c|c|c|c|}
\hline Case & $\begin{array}{c}\text { IHH } \\
(\%)\end{array}$ & $\begin{array}{l}\text { Rehab } \\
\text { center }^{\text {a }}\end{array}$ & $\begin{array}{l}\text { Age } \\
\text { class }^{\mathrm{b}}\end{array}$ & $\operatorname{Sex}^{\mathrm{c}}$ & $\begin{array}{c}\text { Body } \\
\text { mass }(g)\end{array}$ & $\begin{array}{l}\text { Body } \\
\text { score }\end{array}$ & $\mathbf{H t}^{\mathbf{d}}$ & Oil & Moult & $\mathbf{F e}$ & $\begin{array}{c}\text { Concurrent disease and/or } \\
\text { significant lesion }\end{array}$ \\
\hline 1 & 0.4 & IPRAM & $\mathrm{J}$ & $\mathrm{F}$ & 1915 & Thin & N/A & No & No & No & Aspergillosis \\
\hline 2 & 14.8 & IPRAM & $\mathrm{J}$ & $\mathrm{F}$ & 2290 & Thin & N/A & No & No & No & Spleen hemorrhage and rupture \\
\hline 3 & 1.1 & CRAM & $\mathrm{J}$ & M & N/A & N/A & N/A & No & No & N/A & - \\
\hline 4 & 18.3 & R3 & $\mathrm{J}$ & $\mathrm{F}$ & 2560 & Good & $44 \%$ & No & Yes & No & Helminthiasis \\
\hline 5 & 4.2 & $\mathrm{R} 3$ & $\mathrm{~J}$ & M & N/A & Good & $40 \%$ & No & Yes & No & N/A \\
\hline 6 & 5.8 & R3 & $\mathrm{J}$ & M & 3240 & Good & N/A & No & Yes & No & Avipoxvirus \\
\hline 7 & 10.8 & R3 & $\mathrm{J}$ & M & 2400 & N/A & $32 \%$ & No & No & Yes & N/A \\
\hline 8 & 1.5 & $\mathrm{R} 3$ & $\mathrm{~J}$ & ND & 2600 & N/A & N/A & No & No & Yes & $\begin{array}{c}\text { Disseminated intravascular clot- } \\
\text { ting }\end{array}$ \\
\hline 9 & 38.2 & $\mathrm{R} 3$ & $\mathrm{~J}$ & ND & 3450 & N/A & N/A & No & Yes & Yes & N/A \\
\hline 10 & 31.9 & R3 & A & $\mathrm{F}$ & 3300 & Good & N/A & No & No & No & Helminthiasis (small intestine) \\
\hline 11 & 9.4 & R3 & $\mathrm{J}$ & M & 2100 & Good & N/A & No & No & N/A & N/A \\
\hline 12 & 22.8 & IPRAM & $\mathrm{J}$ & $\mathrm{F}$ & 2260 & Good & N/A & No & No & No & $\begin{array}{l}\text { Biliary stasis, Unidentified cysts } \\
\text { (myocardium) }\end{array}$ \\
\hline 13 & 1.4 & CRAM & A & $\mathrm{F}$ & N/A & Good & N/A & No & No & N/A & - \\
\hline 14 & 6.9 & CRAM & A & M & N/A & Good & N/A & No & No & N/A & - \\
\hline 15 & 27.6 & CRAM & $\mathrm{J}$ & $\mathrm{F}$ & 2802 & Good & $50 \%$ & Yes & No & N/A & $\begin{array}{l}\text { Amyloidosis, helminthiasis } \\
\text { (gastrointestinal, lungs, liver) }\end{array}$ \\
\hline 16 & 15 & CRAM & $\mathrm{J}$ & $\mathrm{F}$ & 2810 & Good & $40 \%$ & Yes & No & N/A & Aspergillosis, Amyloidosis \\
\hline 17 & 6.2 & R3 & A & $\mathrm{F}$ & 3220 & Good & N/A & No & No & No & - \\
\hline 18 & 4.8 & R3 & $\mathrm{J}$ & M & 3060 & Good & $27 \%$ & No & Yes & No & Amyloidosis, helminths (air sacs) \\
\hline 19 & 45.4 & $\mathrm{R} 3$ & $\mathrm{~J}$ & $\mathrm{~F}$ & 2700 & Bad & $19 \%$ & No & Yes & No & Aspergillosis, pancarditis \\
\hline 20 & 14.6 & R3 & A & M & 3260 & Good & N/A & No & Yes & No & Helminthiasis \\
\hline 21 & 10.7 & CRAM & $\mathrm{J}$ & ND & 3890 & Good & $36 \%$ & No & No & Yes & - \\
\hline 22 & 12.8 & CRAM & $\mathrm{J}$ & $\mathrm{F}$ & 2910 & N/A & $33 \%$ & No & No & N/A & Spleen hemorrhage and rupture \\
\hline 23 & 8.3 & CRAM & $\mathrm{J}$ & ND & 2645 & Thin & $53 \%$ & Yes & No & Yes & - \\
\hline 24 & 13.8 & CRAM & A & $\mathrm{F}$ & 2750 & Thin & $46 \%$ & Yes & No & Yes & - \\
\hline 25 & 0.9 & IPRAM & J & $\mathrm{F}$ & 1750 & $\begin{array}{l}\text { Cach } \\
\text { ectic }\end{array}$ & N/A & Yes & No & Yes & - \\
\hline 26 & 18.1 & IPRAM & J & $\mathrm{F}$ & 1865 & Thin & N/A & Yes & No & Yes & - \\
\hline 27 & 11.6 & IPRAM & J & $\mathrm{F}$ & 2015 & Thin & N/A & Yes & No & Yes & - \\
\hline 28 & 2.9 & IPRAM & $\mathrm{J}$ & F & 2250 & Thin & N/A & Yes & No & Yes & - \\
\hline 29 & 0.9 & $\mathrm{R} 3$ & J & ND & N/A & Good & N/A & Yes & No & Yes & - \\
\hline 30 & 5.6 & R3 & A & ND & N/A & Good & N/A & Yes & No & Yes & - \\
\hline 31 & 13.4 & CRAM & $\mathrm{J}$ & M & 2248 & N/A & N/A & Yes & No & N/A & - \\
\hline 32 & 6.5 & CRAM & $\mathrm{J}$ & M & 2268 & N/A & N/A & Yes & No & N/A & - \\
\hline 33 & 12.5 & CRAM & $\mathrm{J}$ & M & 3110 & N/A & N/A & Yes & No & N/A & - \\
\hline
\end{tabular}

aIPRAM = Instituto de Pesquisa e Reabilitação de Animais Marinhos (Espírito Santo state, Brazil); R3 = Associaçấo R3 Animal (Santa Catarina state, Brazil); CRAM = Centro de Recuperação de Animais Marinhos (Rio Grande do Sul state, Brazil); ${ }^{b} J=$ Juvenile; $A=$ adult; ${ }^{c} \mathrm{~F}=$ female; $\mathrm{M}=$ male; $\mathrm{ND}=$ not determined; $\mathrm{d} N / \mathrm{A}=$ Information not available.

\section{Results and Discussion}

The pulmonary congestion, intense inflammatory response (especially in liver and spleen), and hemosiderosis found in our birds are in agreement with previous microscopic descriptions of Plasmodium lesions in penguins (FIX et al., 1988; VANSTREELS \& PARSONS, 2014; GRILO et al., 2016). However, there were no significant differences neither in the presence of moderate to severe hepatic hemosiderosis or in $\mathrm{IHH}$ values between
Plasmodium-negative vs. Plasmodium-positive individuals or in regards to the Plasmodium species/lineage. The epidemiology and pathogenicity of the Plasmodium lineages previously diagnosed in our birds (VANSTREELS et al., 2015) raised the question on whether the Plasmodium subgenus Haemamoeba was more pathogenic to these penguins than other Plasmodium subgenera. Unfortunately, we were not able to find a clear answer to that question; these lineages are not completely understood and have been recorded only recently. However, we found higher levels of 
congestion $(\mathrm{p}=0.0182)$ and pneumonia severity $(\mathrm{p}=0.0250)$ between Unidentified lineages vs. Huffia (Figure 2).

Plasmodium-positive females presented significantly higher $\mathrm{IHH}$ values $(17.53 \pm 12.95 \%)$ than males $(7.20 \pm 4.25 \% ; \mathrm{p}=0.041)$ (Figure 3 ), in contrast with findings in toucans and aracaris (Ramphastidae family) (CUBAS, 2008) and in red-spectacled amazons (Amazona pretrei) (Psittacidae family) (PEREIRA et al., 2010), who failed to observe correlation between the rate of hepatic hemosiderosis and sex. Hemosiderosis has been previously associated with egg production (GOTTDENKER et al., 2008), which could not have played a role in our findings based on the age and underdeveloped gonads observed in necropsy. It is not clear why females presented higher IHH values than males; however, one may not eliminate the possibility of sampling influence, either due to the limited number of evaluated birds or higher number of females than males, which reflects previous studies documenting a female-biased sex ratio in Magellanic penguins stranded along the Brazilian coast (VANSTREELS et al., 2013). Comparison between the Pneumonia score (A) and Congestion Score (B) among Plasmodium species/lineages, and IHH in female vs. male Plasmodium-positive Magellanic penguins (Spheniscus magellanicus) (C) are shown in Figure 3.

Concurrent diseases were not statistically relevant. According to the institutions' protocols, many of the birds analyzed in this study were considered anemic, and received iron supplementation, which we believe may have also played a role in the hepatic hemosiderosis observed in this study. The physiological values of circulating iron concentration in Magellanic penguins have been previously evaluated (GHEBREMESKEL et al., 1989); however, to the authors' knowledge, there are no available data regarding iron hepatic levels for this species. Furthermore, the posology of iron supplementation is usually ad-hoc, and might not address the physiological requirements of these birds. In addition, iron represents a critical nutrient for pathogens and tight regulation of iron ("iron-withholding") is a paramount defense strategy of

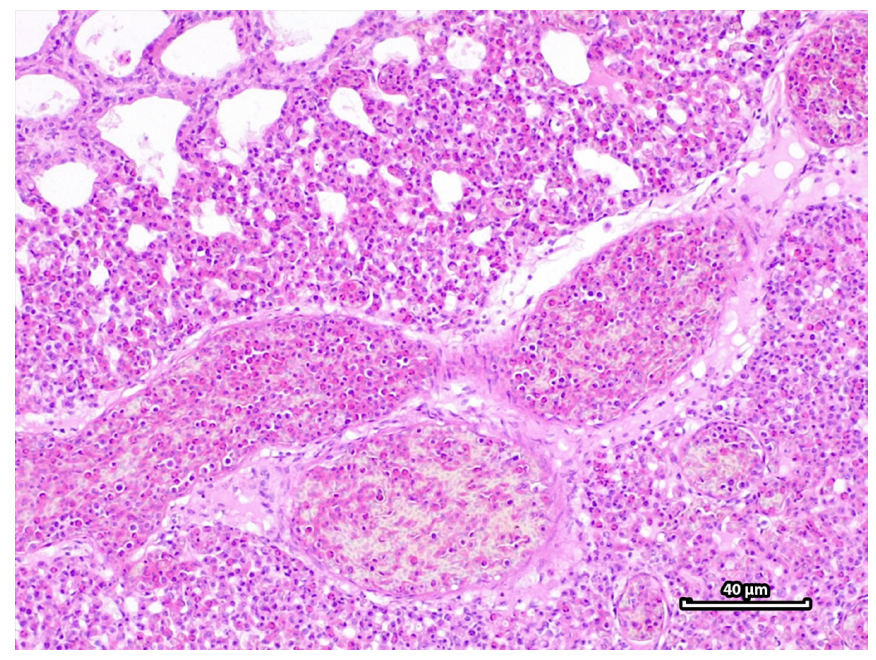

Figure 2. Moderate to severe pulmonary congestion and moderate to severe diffuse granulocytic pneumonia in a Magellanic penguin (Spheniscus magellanicus). H\&E stain. $40 \mu \mathrm{m}$. the host's innate immune system (KOSKI \& SCOTT, 2003; JOHNSON, 2008; BEERNAERT et al., 2010; CASSAT \& SKAAR, 2013). Thus, individuals suffering from iron overload have an enhanced risk of infection (BEERNAERT et al., 2010). Some of our specimens presented gastrointestinal nematodes, air sac parasites, and aspergillosis. It has been suggested that chronic infection or repeated acute infections could lead to greater accumulation of iron in the liver of birds (KLASING et al., 2012). Unfortunately, the low number of concurrent diseases observed in this study prevented statistical evaluation. Aspergillosis and parasitosis could have not only contributed to hemosiderosis, but also thrived upon iron supplementation, further debilitating the affected birds and contributing to their death. Due to all the factors discussed above, we recommend caution in the employment of iron supplementation to Magellanic penguins undergoing rehabilitation.

The IHH values in relation to presence of infection, Plasmodium species/lineage and pathology are summarized in Table 3.

Individual history parameters were not statistically relevant to the IHH values either. Magellanic penguins molt soon after breeding season, immediately prior to their winter migration (SILVA-FILHO \& RUOPPOLO, 2014). Anseriformes and Passeriformes of the Sturnidae family showed an association between the hepatic level of hemosiderosis in this same period, with increased amounts of positive iron staining primarily located within lysosomic organelles of parenchymal hepatic cells (WARD et al., 1988; CORK et al., 1995). Such findings are possibly related to an increased hematopoietic activity and consequent erythrocyte production required to enhance oxygen uptake during periods of increased thermogenesis or changes in the levels of thyroid hormone involved in the molting process (WARD et al., 1988; CORK et al., 1995; OSBORN, 1979; CORK, 2000). Although not statistically relevant, some birds $(4,5,6,9,18,19$, and 20) were molting during the study, so it is not possible to completely discard the hypothesis that residual iron from molting might have contributed to the hepatic hemosiderosis observed in our birds.

Fat can be severely depleted during migration, forcing birds to use body protein from the heart, pectoral, and leg muscles and the digestive organs (intestine, gizzard, and liver) as a second fuel option (LINDSTRÖM \& PIERSMA, 1993; BATTLEY et al., 2000; BAUCHINGER \& BIEBACH, 2001; SCHWILCH et al., 2002; MCWILLIAMS et al., 2004). Protein metabolism stems to a large extent from the skeletal muscles, which in penguins are especially rich in myoglobin, releasing the iron present in heme group of myoglobin, and possibly contributing to the development of hemosiderosis (SCHWARTZ et al., 1973; BAUCHINGER \& BIEBACH, 2001; PONGANIS et al., 2010). The majority of migrating Magellanic penguins stranded in Brazil are in poor body condition, with markedly atrophied skeletal muscles and no subcutaneous fat deposits (CARDOSO et al., 2011). We assessed the true nutritional condition of these birds: body mass (ranging from $3.5-5 \mathrm{~kg}$ in wild specimens) and body condition score (SILVA-FILHO \& RUOPPOLO, 2014). The only 2 emaciated birds (1 and 19), however, presented $\mathrm{IHH}$ values within the range found in this study. Nevertheless, increased iron deposition has been reported in malnourished black-necked swans (Cygnus melanocoryphus) (NORAMBUENA \& BOZINOVIC, 2009), in the liver of rats 

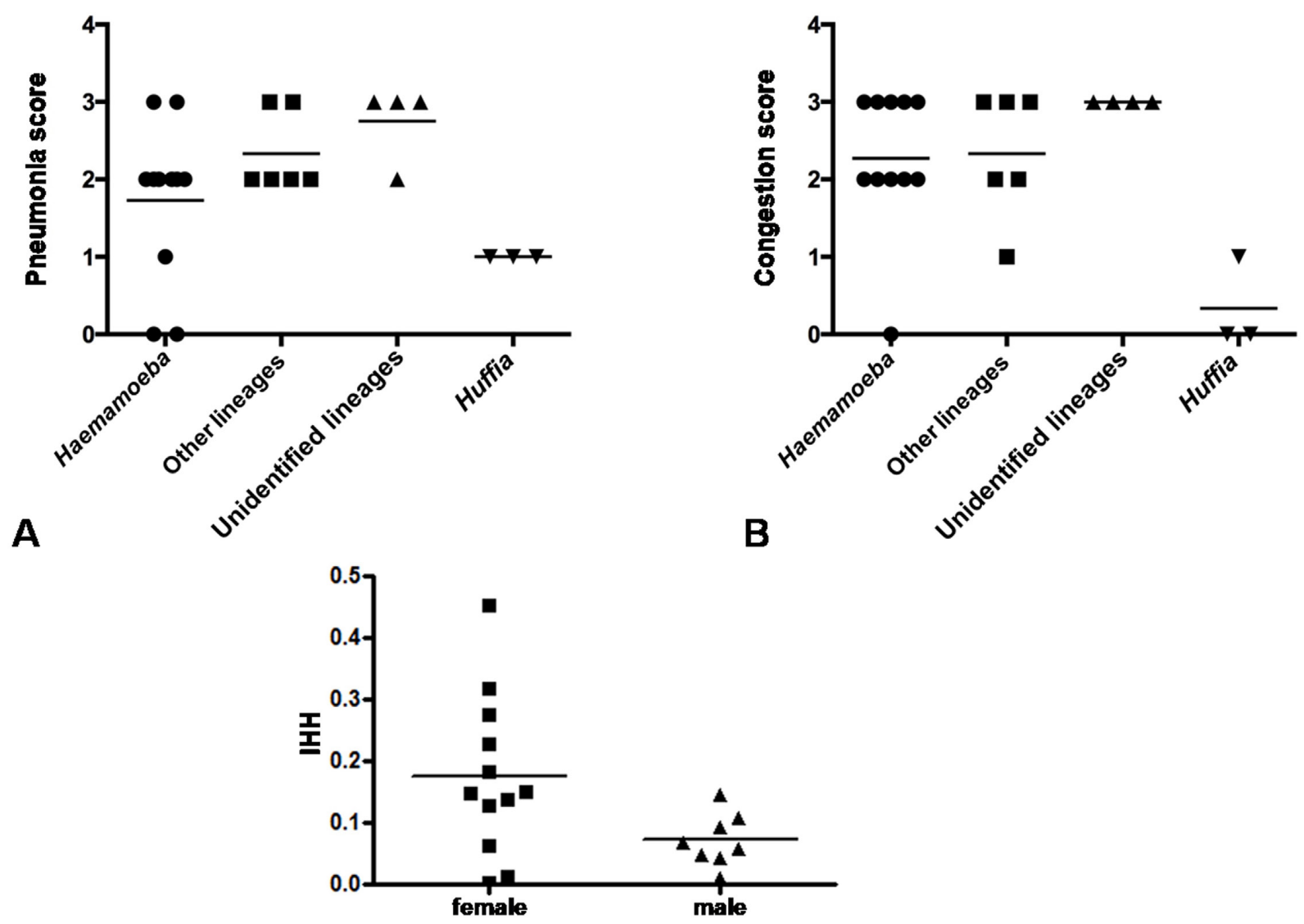

C

Figure 3. Comparison between the Pneumonia score (A) and Congestion Score (B) among Plasmodium species/lineages, and the index of hepatic hemosiderosis (IHH) in female vs. male Plasmodium-positive Magellanic penguins (Spheniscus magellanicus) (C).

subjected to food restriction (ROZENTAL et al., 1967), and in some severe cases of prolonged malnutrition in obese humans subjected to a variety of fasting periods (MITCHELL \& JOHNS, 2008). It is important to consider that malnutrition may have a role in hemosiderosis in migratory bird species.

Intoxication (e.g., petroleum products) and hemoparasitosis are known causes of hemolytic anemia (FRY \& LOWENSTINE, 1985). Systemic absorption of petroleum and its derivatives following acute or chronic oil exposure causes hemolytic anemia in birds (LEIGHTON, 1986; KHAN \& RYAN, 1991; DINHOPL et al., 2011). Oil pollution is an important conservation threat to Magellanic penguins on wintering grounds (GARCÍA-BORBOROGLU et al., 2006). In this study, however, IHH was not significantly different between individuals with and without history of acute oil contamination upon arrival at the rehabilitation centers, suggesting that (a) oil exposure may have been too superficial or rapidly mitigated and therefore systemic absorption was minimal, and/or (b) other factors may have played a greater role in producing/modulating hemosiderosis in a manner that surpassed or contradicted any detectable effects from oiling. Nevertheless, these birds' history during migration is unknown, so we were not able to completely exclude the potential influence of chronic oil exposure in the hepatic hemosiderosis seen in this study.
Although previous studies (CRANFIELD et al., 1994; ONG et al., 2006) suggested that the low parasitaemia levels seen in Plasmodium-infected penguins are not sufficient to cause clinical anemia, Plasmodium-infected erythrocytes may suffer hemolysis or induce erythrocyte sequestration and extravascular hemolysis (VALKIŪNAS, 2005), releasing iron, which is subsequently stored in macrophages, as ferritin and hemosiderin (RATLEDGE, 2007). Eventually, cases 2 and 22 presented spleen hemorrhage and rupture, whereas case 8 presented disseminated intravascular coagulation - acute vascular complications likely associated with avian malaria that if not the primary cause, contributed to the development of secondary hemosiderosis. Another possible cause of anemia was the infestation by gastrointestinal nematodes (cases 4, 10, 15, 18 and 20).

Liver mass changes caused by the spontaneous long-term fasting characteristic of the annual cycle of penguins during reproduction and molt (GROSCOLAS \& ROBIN, 2001) and/or due to concurrent diseases could have interfered with the morphometric evaluation of hepatic hemosiderin. It is unclear if changes in digestive organs of birds during migration are related to cell size (hypertrophy/atrophy) and/or cell number (hyperplasia/hypoplasia) (WAGENMAKERS et al., 1984). Further morphometric studies are needed in order to test these possibilities. 
Both the amount of dietary iron and length of exposure to dietary iron are factors in hepatic iron accumulation in birds (CRISSEY et al., 2000; HELMICK et al., 2011). Unfortunately, data regarding their diet while in the Brazilian continental shelf during the winter migration is limited and there is no such information regarding the species offered to the studied birds while under care. To our knowledge, the only suggested nutritional iron concentration for adult penguin diets in a dry matter basis $(60-80 \mathrm{mg} / \mathrm{kg})$ is based on requirements of domestic poultry, cats, and inferences from wild foods composition (WALLACE \& WALSH, 2005; AZA Penguin Taxon Advisory Group, 2014). Further studies are needed to determine the nutritional iron requirements of Magellanic penguins.

Finally, our findings show that other factors aside from avian malaria likely played a role in the presence of hepatic hemosiderosis in Plasmodium-infected and Plasmodium-negative Magellanic penguins under rehabilitation in Brazil, and the importance of considering the species life cycle and clinical history when evaluating hepatic hemosiderosis. We propose that the increased hepatic hemosiderosis noted in this study was multifactorial, the result of pathological processes caused by malaria, molting, hemoglobin and myoglobin catabolism promoted by the intense energetic demands of Magellanic penguins' winter migration, anemia, concomitant diseases, and iron supplementation while under care, all possibly potentiated by an altered liver mass.

\section{Acknowledgements}

We thank the staffs of the Centro de Recuperação de Animais Marinhos da Universidade Federal do Rio Grande (CRAM-FURG), Associação R3 Animal and Instituto de Pesquisa e Reabilitação de Animais Marinhos (IPRAM), for their collaboration and support, and the State Environmental and Water Resources Institute (IEMA) and Brazilian Institute of the Environment and Renewable Natural Resources (IBAMA) for their assistance with the penguins rescued in Espírito Santo. We also thank Jorge Oyakawa, Sândara Sguario and Luciano Bugalho at the School of Veterinary Medicine and Animal Sciences, University of São Paulo for their technical support. This study was supported by the Coordination for the Improvement of Higher Level Personnel (CAPES) and the São Paulo Research Foundation (FAPESP) under Grant [2009/53956-9, 2010/51801-5].

\section{References}

AZA Penguin Taxon Advisory Group. Penguin (Spheniscidae) care manual [online]. Silver Spring: Association of Zoos and Aquariums; 2014 [cited 2018 Mar 30]. Available from: https:/www.speakcdn.com/assets/2332/ penguin_care_manual_aza_final_2014.pdf

Bak UB, Park JC, Lim YJ. An outbreak of malaria in penguins at the Farm-land Zoo. Korean J Parasitol 1984; 22(2): 267-272. http://dx.doi. org/10.3347/kjp.1984.22.2.267. PMid:12891023.

Baldassin P, Taniguchi S, Gallo H, Silva RJ, Montone RC. Persistent organic pollutants in juvenile Magellan penguins (Spheniscus magellanicus) found on the northern shore of the state of São Paulo and southern shore of the state of Rio de Janeiro, Brazil. Mar Pollut Bull 2012; 64(11): 2502-2506. http://dx.doi.org/10.1016/j.marpolbul.2012.07.035. PMid:22910330.

Battley PF, Piersma T, Dietz MW, Tang S, Dekinga A, Hulsman K. Empirical evidence for differential organ reductions during trans-oceanic bird flight. Proc Biol Sci 2000; 267(1439): 191-195. http://dx.doi. org/10.1098/rspb.2000.0986. PMid:10687826.

Bauchinger U, Biebach $\mathrm{H}$. Differential catabolism of muscle protein in garden warblers (Sylvia borin): flight and leg muscle act as a protein source during long-distance migration. J Comp Physiol B 2001; 171(4): 293-301. http://dx.doi.org/10.1007/s003600100176. PMid:11409626.

Beernaert LA, Pasmans F, Van Waeyenberghe L, Haesebrouck F, Martel A. Aspergillus infections in birds: a review. Avian Pathol 2010; 39(5): 325-331. http://dx.doi.org/10.1080/03079457.2010.506210. PMid:20954008.

Brandão ML, Braga KM, Luque JL. Marine debris ingestion by Magellanic penguins, Spheniscus magellanicus (Aves: Sphenisciformes), from the Brazilian coastal zone. Mar Pollut Bull 2011; 62(10): 2246-2249. http:// dx.doi.org/10.1016/j.marpolbul.2011.07.016. PMid:21864861.

Bueno MG, Lopez RPG, Menezes RMT, Costa-Nascimento MJ, Lima GFMC, Araújo RAS, et al. Identification of Plasmodium relictum causing mortality in penguins (Spheniscus magellanicus) from São Paulo Zoo, Brazil. Vet Parasitol 2010; 173(1-2): 123-127. http://dx.doi.org/10.1016/j. vetpar.2010.06.026. PMid:20638795.

Cardoso LG, Bugoni L, Mancini PL, Haimovici M. Gillnet fisheries as a major mortality factor of Magellanic penguins in wintering areas. Mar Pollut Bull 2011; 62(4): 840-844. http://dx.doi.org/10.1016/j. marpolbul.2011.01.033. PMid:21376348.

Cassat JE, Skaar EP. Iron in infection and immunity. Cell Host Microbe 2013; 13(5): 509-519. http://dx.doi.org/10.1016/j.chom.2013.04.010. PMid:23684303.

Cork SC, Alley MR, Stockdale PHG. A quantitative assessment of haemosiderosis in wild and captive birds using image analysis. Avian Pathol 1995; 24(2): 239-254. http://dx.doi.org/10.1080/03079459508419066. PMid:18645783.

Cork SC. Iron storage diseases in birds. Avian Pathol 2000; 29(1): 7-12. http://dx.doi.org/10.1080/03079450094216. PMid:19184782.

Cranfield MR, Graczyk TK, Beall FB, laleggio DM, Shaw ML, Skjoldager ML. Subclinical avian malaria infections in African black-footed penguins (Spheniscus demersus) and induction of parasite recrudescence. JWildl Dis 1994; 30(3): 372-376. http://dx.doi.org/10.7589/0090-3558-30.3.372. PMid:7933280.

Crissey SD, Ward AM, Block SE, Maslanka MT. Hepatic iron accumulation over time in European starlings (Sturnus vulgaris) fed two levels of iron. J Zoo Wildl Med 2000; 31(4): 491-496. http://dx.doi.org/10.1638/10427260(2000)031 [0491:HIAOTI]2.0.CO;2. PMid:11428395.

Cubas ZS. Siderose hepática em tucanos e araçaris [dissertação]. Paraná: Universidade Federal do Paraná; 2008.

Dinhopl N, Mostegl MM, Richter B, Nedorost N, Maderner A, Fragner $\mathrm{K}$, et al. Application of in-situ hybridization for the detection and identification of avian malaria parasites in paraffin wax-embedded tissues from captive penguins. Avian Pathol 2011; 40(3): 315-320. http://dx.doi. org/10.1080/03079457.2011.569533. PMid:21711191.

Fantham HB, Porter A. On a Plasmodium (Plasmodium relictum var. spheniscidae, n. var.), observed in four species of penguins. J Zool 1944; 114(3): 279-292. 
Fix AS, Waterhouse C, Greiner EC, Stoskopf MF. Plasmodium relictum as a cause of avian malaria in wild-caught Magellanic penguins (Spheniscus magellanicus). J Wildl Dis 1988; 24(4): 610-619. http://dx.doi. org/10.7589/0090-3558-24.4.610. PMid:3193555.

Fry DM, Lowenstine LJ. Pathology of common murres and Cassin's auklets exposed to oil. Arch Environ Contam Toxicol 1985; 14(6): 725 737. http://dx.doi.org/10.1007/BF01055780.

García-Borboroglu P, Boersma PD, Ruoppolo V, Pinho-da-Silva-Filho R, Corrado-Adornes A, Conte-Sena D, et al. Magellanic penguin mortality in 2008 along the SW Atlantic coast. Mar Pollut Bull 2010; 60(10): 1652-1657. http://dx.doi.org/10.1016/j.marpolbul.2010.07.006. PMid:20674946.

García-Borboroglu P, Boersma PD, Ruoppolo V, Reyes L, Rebstock GA, Griot K, et al. Chronic oil pollution harms Magellanic penguins in the Southwest Atlantic. Mar Pollut Bull 2006; 52(2): 193-198. http://dx.doi. org/10.1016/j.marpolbul.2005.11.004. PMid:16364372.

Ghebremeskel K, Williams G, Keymer IF, Horsley D, Gardner DA. Plasma chemistry of rockhopper (Eudyptes crestatus), Magellanic (Spheniscus magellanicus) and gentoo (Pygoscelis papua) wild penguins in relation to moult. Comp Biochem Physiol A Physiol 1989; 92(1): 43-47.

Gottdenker NL, Walsh T, Jiménez-Uzcátegui G, Betancourt F, Cruz M, Soos C, et al. Causes of mortality of wild birds submitted to the Charles Darwin Research Station, Santa Cruz, Galapagos, Ecuador from 2002-2004. J Wildl Dis 2008; 44(4): 1024-1031. http://dx.doi. org/10.7589/0090-3558-44.4.1024. PMid:18957662.

Grilo ML, Vanstreels RET, Wallace R, García-Párraga D, Braga ÉM, Chitty J, et al. Malaria in penguins - current perceptions. Avian Pathol 2016; 45(4): 393-407. http://dx.doi.org/10.1080/03079457.2016.114 9145. PMid:27009571.

Griner LA, Sheridan BW. Malaria (Plasmodium relictum) in penguins at the San Diego Zoo. Am J Vet Clin Pathol 1967; 1: 7-17.

Groscolas R, Robin JP. Long-term fasting and re-feeding in penguins. Comp Biochem Physiol A Mol Integr Physiol 2001; 128(3): 645-653. http://dx.doi.org/10.1016/S1095-6433(00)00341-X. PMid:11246051.

Helmick KE, Kendrick EL, Dierenfeld ES. Diet manipulation as treatment for elevated serum iron parameters in captive Raggiana bird of paradise (Paradisaea raggiana). J Zoo Wildl Med 2011; 42(3): 460-467. http:// dx.doi.org/10.1638/2010-0131.1. PMid:22950319.

Johnson L. Iron and siderophores in fungal-host interactions. Mycol Res 2008; 112(2): 170-183. http://dx.doi.org/10.1016/j.mycres.2007.11.012. PMid:18280720.

Jones HI, Shellam GR. Blood parasites in penguins, and their potential impact on conservation. Mar Ornithol 1999; 27: 181-184.

Kehrig HA, Hauser-Davis RA, Seixas TG, Fillmann G. Trace-elements, methylmercury and metallothionein levels in Magellanic penguin (Spheniscus magellanicus) found stranded on the Southern Brazilian coast. Mar Pollut Bull 2015; 96(1-2): 450-455. http://dx.doi.org/10.1016/j. marpolbul.2015.05.006. PMid:25960272.

Khan RA, Ryan P. Long term effects of crude oil on common murres (Uria aalge) following rehabilitation. Bull Environ Contam Toxicol 1991; 46(2): 216-222. http://dx.doi.org/10.1007/BF01691940. PMid:2018868.

Klasing KC, Dierenfeld ES, Koutsos EA. Avian iron storage disease: variations on a common theme? J Zoo Wildl Med 2012;43(3 Suppl): 27-34. http://dx.doi.org/10.1638/2011-0157.1. PMid:23156703.
Koski KG, Scott ME. Gastrointestinal nematodes, trace elements, and immunity. J Trace Elem Exp Med 2003; 16(4): 237-251. http://dx.doi. org/10.1002/jtra.10043.

Laird M. Some blood parasites of New Zealand birds. Zool Pub Victoria Uni College. 1950; 5: 1-20.

Leighton FA. Clinical, gross, and histological findings in herring gulls and Atlantic puffins that ingested Prudhoe Bay crude oil. Vet Pathol 1986; 23(3): 254-263. http://dx.doi.org/10.1177/030098588602300305. PMid:3727312.

Levin II, Outlaw DC, Vargas FH, Parker PG. Plasmodium blood parasite found in endangered Galapagos penguins (Spheniscus mendiculus). Biol Conserv 2009; 142(12): 3191-3195. http://dx.doi.org/10.1016/j. biocon.2009.06.017.

Lindström $\AA$, Piersma T. Mass changes in migrating birds: the evidence for fat and protein storage re-examined. Ibis 1993; 135(1): 70-78. http:// dx.doi.org/10.1111/j.1474-919X.1993.tb02811.x.

Lowenstein LJ, Munson L. Iron overload in the animal kingdom. In: Fowler ME, Miller RE. Zoo and wild animal medicine - current therapy 4. Philadelphia: WB Saunders; 1999. p. 260-269.

Luna LG. Histopathological methods and color atlas of special stains and tissue artifacts. Gaithersburg: American Histolabs; 1992.

McWilliams SR, Guglielmo C, Pierce B, Klaassen M. Flying, fasting, and feeding in birds during migration: a nutritional and physiological ecology perspective. J Avian Biol 2004; 35(5): 377-393. http://dx.doi. org/10.1111/j.0908-8857.2004.03378.x.

Mitchell EB, Johns J. Avian hematology and related disorders. Vet Clin North Am Exot Anim Pract 2008; 11(3): 501-522, vi. http://dx.doi. org/10.1016/j.cvex.2008.03.004. PMid:18675731.

Norambuena MC, Bozinovic F. Effect of malnutrition on iron homeostasis in black-necked swans (Cygnus melanocoryphus). J Zoo Wildl Med 2009; 40(4): 624-631. http://dx.doi.org/10.1638/2007-0160.1. PMid:20063807.

Ong ST, Ho JZS, Ho B, Ding JL. Iron-withholding strategy in innate immunity. Immunobiology 2006; 211(4): 295-314. http://dx.doi. org/10.1016/j.imbio.2006.02.004. PMid:16697921.

Osborn D. Seasonal changes in the fat, protein and metal content of the liver of the starling Sturnus vulgaris. Environ Pollut 1979; 19(2): 145-155. http://dx.doi.org/10.1016/0013-9327(79)90145-9.

Parsons NJ, Underhill LG. Oiled and injured African penguins Spheniscus demersus and other seabirds admitted for rehabilitation in the Western Cape, South Africa, 2001 and 2002. Afr J Mar Sci 2005; 27(1): 289-296. http://dx.doi.org/10.2989/18142320509504087.

Pereira LQ, Strefezzi RDF, Catão-Dias JL, Trindade MM, Fighera RA, Kommers GD, et al. Hepatic hemosiderosis in red-spectacled amazons (Amazona pretrei) and correlation with nutritional aspects. Avian Dis 2010; 54(4): 1323-1326. http://dx.doi.org/10.1637/9418-060710Case.1. PMid:21313859.

Petry MV, Fonseca VSS. Effects of human activities in the marine environment on seabirds along the coast of Rio Grande do Sul, Brazil. Ornitol Neotrop 2002; 13: 137-142.

Ponganis PJ, Welch TJ, Welch LS, Stockard TK. Myoglobin production in emperor penguins. J Exp Biol 2010; 213(11): 1901-1906. http:// dx.doi.org/10.1242/jeb.042093. PMid:20472777. 
Ratledge C. Iron metabolism and infection. Food Nutr Bull 2007;28(4 Suppl): 515-523. http://dx.doi.org/10.1177/15648265070284S405. PMid:18297890.

Rezende GC, Baldassin P, Gallo H, Silva RJ. Ecological aspects of helminth fauna of Magellanic penguins, Spheniscus magellanicus (Aves: Spheniscidae), from the northern coast of the State of São Paulo, Brazil. Braz J Biol 2013; 73(1): 61-66. http://dx.doi.org/10.1590/S151969842013000100008. PMid:23644789.

Rodrigues SC, Adornes AC, Santos Filho EC, Silva-Filho RP, Colares EP. Surviving probability indicators of landing juvenile Magellanic penguins arriving along the southern Brazilian coast. Braz Arch Biol Technol 2010; 53(2): 419-424. http://dx.doi.org/10.1590/S1516-89132010000200021.

Rozental P, Biava C, Spencer H, Zimmerman HJ. Liver morphology and function tests in obesity and during total starvation. Am J Dig Dis 1967; 12(2): 198-208. http://dx.doi.org/10.1007/BF02233443. PMid:6016689.

Ruoppolo V, Vanstreels RET, Woehler EJ, Heredia SA, Adornes AC, Silva-Filho RP, et al. Survival and movements of Magellanic penguins rehabilitated from oil fouling along the coast of South America, 2000-2010. Mar Pollut Bull 2012; 64(7): 1309-1317. http://dx.doi.org/10.1016/j. marpolbul.2012.05.009. PMid:22683106.

Schwartz E, Tornaben JA, Boxill GC. The effects of food restriction on hematology, clinical chemistry and pathology in the albino rat. Toxicol Appl Pharmacol 1973; 25(4): 515-524. http://dx.doi.org/10.1016/0041008X(73)90020-3. PMid:4741773.

Schwilch R, Grattarola A, Spina F, Jenni L. Protein loss during longdistance migratory flight in passerine birds: adaptation and constraint. J Exp Biol 2002; 205(5): 687-695. PMid:11907058.

Sheppard C, Dierenfeld E. Iron storage disease in birds: speculation on etiology and implications for captive husbandry. J Avian Med Surg 2002; 16(3): 192-197. http://dx.doi.org/10.1647/1082-6742(2002)016[0192:IS DIBS]2.0.CO;2.

Silva-Filho RP, Ruoppolo V. Sphenisciformes (Pinguim). In: Cubas ZS, Silva JCR, Catâo-Dias JL. Tratado de Animais Selvagens. 2nd ed. São Paulo: Roca; 2014. p. 384-416.

Silveira P, Belo NO, Lacorte GA, Kolesnikovas CK, Vanstreels RE, Steindel M, et al. Parasitological and new molecular-phylogenetic characterization of the malaria parasite Plasmodium tejerai in South
American penguins. Parasitol Int 2013; 62(2): 165-171. http://dx.doi. org/10.1016/j.parint.2012.12.004. PMid:23269202.

Stokes DL, Boersma PD, Casenave JL, García-Borboroglu P. Conservation of migratory Magellanic penguins requires marine zoning. Biol Conserv 2014; 170: 151-161. http://dx.doi.org/10.1016/j.biocon.2013.12.024.

Valkiūnas G. Avian malaria parasites and other haemosporidia. Boca Raton: CRC Press; 2005.

Vanstreels RET, Adornes AC, Canabarro PL, Ruoppolo V, Amaku M, Silva-Filho RP, et al. Female-biased mortality of Magellanic Penguins (Spheniscus magellanicus) on the wintering grounds. Emu 2013; 113(2): 128-134. http://dx.doi.org/10.1071/MU12060.

Vanstreels RET, Braga EM, Catão-Dias JL. Blood parasites of penguins: a critical review. Parasitology 2016; 143(8): 931-956. http://dx.doi. org/10.1017/S0031182016000251. PMid:27253438.

Vanstreels RET, Kolesnikovas CK, Sandri S, Silveira P, Belo NO, Ferreira FC Jr, et al. Outbreak of avian malaria associated to multiple species of Plasmodium in Magellanic penguins undergoing rehabilitation in Southern Brazil. PLoS One 2014; 9(12): e116554. http://dx.doi.org/10.1371/ journal.pone.0116554. PMid:24736326.

Vanstreels RET, Parsons NJ. Malária aviária e outros Hemosporídeos aviários. In: Cubas ZS, Silva JCR, Catão-Dias JL. Tratado de animais selvagens. 2nd ed. Sáo Paulo: Roca; 2014. p. 1427-1443.

Vanstreels RET, Silva-Filho RP, Kolesnikovas CKM, Bhering RCC, Ruoppolo V, Epiphanio S, et al. Epidemiology and pathology of avian malaria in penguins undergoing rehabilitation in Brazil. Vet Res 2015; 46(1): 30. http://dx.doi.org/10.1186/s13567-015-0160-9. PMid:25888987.

Wagenmakers AJ, Schepens JT, Veerkamp JH. Effect of starvation and exercise on actual and total activity of the branched-chain 2-oxo acid dehydrogenase complex in rat tissues. Biochem J 1984; 223(3): 815-821. http://dx.doi.org/10.1042/bj2230815. PMid:6508743.

Wallace R, Walsh M. Health. In: Penguin Taxon Advisory Group. Penguin Husbandry Manual. 3rd ed. Silver Spring: American Zoo and Aquarium Association; 2005. p. 86-111.

Ward RJ, Iancu TC, Henderson GM, Kirkwood JR, Peters TJ. Hepatic iron overload in birds: analytical and morphological studies. Avian Pathol 1988; 17(2): 451-464. http://dx.doi.org/10.1080/03079458808436463. PMid:18766702. 